\title{
Boundedness of Pseudo-Differential Operators on $\boldsymbol{L}^{p}$, Sobolev and Modulation Spaces
}

\author{
S. Molahajloo ${ }^{1 *}$, G.E. Pfander ${ }^{2}$ \\ ${ }^{1}$ Department of Mathematics and Statistics, Queen's University, Kingston, Ontario K7L3N6, Canada \\ ${ }^{2}$ School of Engineering and Science, Jacobs University, 28759 Bremen, Germany
}

\begin{abstract}
We introduce new classes of modulation spaces over phase space. By means of the Kohn-Nirenberg correspondence, these spaces induce norms on pseudo-differential operators that bound their operator norms on $L^{p}$-spaces, Sobolev spaces, and modulation spaces.
\end{abstract}

Keywords and phrases: pseudo-differential operators, modulation spaces, Sobolev spaces, short-time Fourier transforms

Mathematics Subject Classification: 47G30, 42B35, 35S05, 46E35

\section{Introduction}

Pseudo-differential operators are discussed in various areas of mathematics and mathematical physics, for example, in partial differential equations, time-frequency analysis, and quantum mechanics $[18,19,21$, 32,34]. They are defined as follows.

Let $\sigma$ be a tempered distribution on phase space $\mathbb{R}^{2 d}$, that is, $\sigma \in \mathcal{S}^{\prime}\left(\mathbb{R}^{2 d}\right)$ where $\mathcal{S}\left(\mathbb{R}^{2 d}\right)$ denotes the space of Schwartz class functions. The pseudo-differential operator $T_{\sigma}$ corresponding to the symbol $\sigma$ is given by

$$
T_{\sigma} f(x)=\int \sigma(x, \xi) \widehat{f}(\xi) e^{2 \pi i x \cdot \xi} d \xi, \quad f \in \mathcal{S}\left(\mathbb{R}^{d}\right) .
$$

Here, $\widehat{f}$ denotes the Fourier transform of $f$, namely,

$$
\widehat{f}(\xi)=\mathcal{F} f(\xi)=\int f(x) e^{-2 \pi i x \cdot \xi} d x .
$$

One of the central goals in the study of pseudo-differential operators is to obtain necessary and sufficient conditions for pseudo-differential operators to extend boundedly to function spaces such as $L^{p}\left(\mathbb{R}^{d}\right)[3,5$, 20,33]. A classical result in this direction is the following.

For $m \in \mathbb{R}$, we let $S^{m}$ consist of all functions $\sigma$ in $C^{\infty}\left(\mathbb{R}^{d} \times \mathbb{R}^{d}\right)$ such that for any multi-index $(\alpha, \beta)$ there is $C_{\alpha, \beta}>0$ with

$$
\left|\left(\partial_{x}^{\beta} \partial_{\xi}^{\alpha} \sigma\right)(x, \xi)\right| \leq C_{\alpha, \beta}(1+|\xi|)^{m-|\alpha|} .
$$

For $\sigma \in S^{0}\left(\mathbb{R}^{d}\right)$ it is known that $T_{\sigma}$ acts boundedly on $L^{p}\left(\mathbb{R}^{d}\right), p \in(1, \infty)$. A consequence of this result is that if $\sigma \in S^{m}$, then $T_{\sigma}$ is a bounded operator mapping $H_{s+m}^{p}\left(\mathbb{R}^{d}\right)$ to $H_{s}^{p}\left(\mathbb{R}^{d}\right)$, where $H_{s}^{p}\left(\mathbb{R}^{d}\right)$ is the

\footnotetext{
*Corresponding author. E-mail: molahajloo@mast.queensu.ca
} 
Sobolev Spaces of order $s \in \mathbb{R}$; for more details see Wong's book [32]. Similarly, in [33], Wong obtains weighted $L^{p}$-boundedness results for pseudo-differential operators with symbols in $S^{m}$.

Smoothness and boundedness of symbols though are far from being necessary (nor sufficient) for the $L^{p}$-boundedness of pseudo-differential operators. In fact, every symbol $\sigma \in L^{2}\left(\mathbb{R}^{2 d}\right)$ defines a so-called Hilbert-Schmidt operator and Hilbert-Schmidt operators are bounded, in fact, compact operators on $L^{2}\left(\mathbb{R}^{d}\right)$. Non-smooth and unbounded symbols have been considered systematically in the framework of modulation spaces, an approach that we follow in this paper.

Modulation spaces were first introduced by Feichtinger in [9] and they have been further developed by him and Gröchenig in [8-13]. In the following, set $\phi(x)=e^{-\pi\|x\|^{2} / 2}$ and let the dual pair bracket $(\cdot, \cdot)$ be linear in the first argument and antilinear in the second argument.

Definition 1.1 (Modulation spaces over Euclidean space). Let $M_{\nu}$ denote modulation by $\nu \in \mathbb{R}^{d}$, namely, $M_{\nu} f(x)=e^{2 \pi i t \cdot \nu} f(x)$, and let $T_{t}$ be translation by $t \in \mathbb{R}^{d}$, that is, $T_{t} f(x)=g(x-t)$.

The short-time Fourier transform $V_{\phi} f$ of $f \in \mathcal{S}^{\prime}\left(\mathbb{R}^{d}\right)$ with respect to the Gaussian window $\phi$ is given by

$$
V_{\phi} f(t, \nu)=\mathcal{F}\left(f T_{t} \phi\right)(\nu)=\left(f, M_{\nu} T_{t} \phi\right)=\int f(x) e^{-2 \pi i x \nu} \phi(x-t) d x
$$

The modulation space $M^{p q}\left(\mathbb{R}^{d}\right), 1 \leq p, q \leq \infty$, is a Banach space consisting of those $f \in \mathcal{S}^{\prime}\left(\mathbb{R}^{d}\right)$ with

$$
\|f\|_{M^{p q}}=\left\|V_{\phi} f\right\|_{L^{p q}}=\left(\int\left(\int\left|V_{\phi} f(t, \nu)\right|^{p} d t\right)^{1 / p} d \nu\right)^{1 / q}<\infty
$$

with usual adjustment if $p=\infty$ and/or $q=\infty$.

Roughly speaking, distributions in $M^{p q}\left(\mathbb{R}^{d}\right)$ 'decay' at infinity like a function in $L^{p}\left(\mathbb{R}^{d}\right)$ and have the same local regularity as a function whose Fourier transform is in $L^{q}\left(\mathbb{R}^{d}\right)$.

The boundedness of pseudo-differential operators on modulation spaces are studied for various classes of symbols, for example, in $[5,7,15,16,27,28,30,31]$. In $[27,28]$ for example, Toft discusses boundedness of pseudo-differential operators on weighted modulation spaces. In [5], Nicola and Cordero describe a class of pseudo-differential operators with symbols $\sigma$ in modulation spaces for which $T_{\sigma}$ is bounded on $L^{p}\left(\mathbb{R}^{d}\right)$.

The modulation space membership criteria on Kohn-Nirenberg symbols used in $[5,7,27,28]$ do not allow to require different decays in $x$ and $\xi$ of $\sigma(x, \xi)$. In the recently developed sampling theory for operators, though, a separate treatment of the decay in $x$ and $\xi$ was beneficial $[17,23,24]$. In fact, this allows to realize canonical symbol norms of convolution and multiplication operators as modulation space norms on Kohn-Nirenberg symbols. Motivated by this work, we give the following definition.

Definition 1.2 (Modulation spaces over phase space). The symplectic Fourier transform of $F \in \mathcal{S}\left(\mathbb{R}^{2 d}\right)$ is given by

$$
\widetilde{\mathcal{F}} F(t, \nu)=\int_{\mathbb{R}^{2 d}} e^{-2 \pi i[(x, \xi),(t, \nu)]} F(x, \xi) d x d \xi,
$$

where $[(x, \xi),(t, \nu)]$ is the symplectic form of $(x, \xi)$ and $(t, \nu)$ defined by $[(x, \xi),(t, \nu)]=x \cdot \nu-\xi \cdot t$. Analogously, symplectic modulation $\widetilde{M}_{(t, \nu)}$ is defined by $\widetilde{M}_{(t, \nu)} F(x, \xi)=e^{2 \pi i[(x, \xi),(t, \nu)]} F(x, \xi)$.

The symplectic short-time Fourier transform $\widetilde{V}_{\phi} f$ of $F \in \mathcal{S}^{\prime}\left(\mathbb{R}^{d}\right)$ is then given by

$$
\begin{aligned}
\widetilde{V}_{\phi} F(x, t, \xi, \nu) & =\widetilde{\mathcal{F}}\left(F T_{(x, \xi)} \phi\right)(t, \nu)=\left(F, \widetilde{M}_{(\nu, t)} T_{(x, \xi)} \phi\right) \\
& =\iint e^{-2 \pi i(\widetilde{x} \nu-\widetilde{\xi} t)} F(\widetilde{x}, \widetilde{\xi}) \varphi(\widetilde{x}-x, \widetilde{\xi}-\xi) d \widetilde{x} d \widetilde{\xi}
\end{aligned}
$$


The modulation space over phase space $\widetilde{M}^{p_{1} p_{2} q_{1} q_{2}}\left(\mathbb{R}^{2 d}\right), 1 \leq p_{1}, p_{2}, q_{1}, q_{2} \leq \infty$, is the Banach space consisting of those $F \in \mathcal{S}^{\prime}\left(\mathbb{R}^{2 d}\right)$ with

$$
\begin{aligned}
& \|F\|_{\widetilde{M}^{p_{1} p_{2} q_{1} q_{2}}}=\left\|\widetilde{V}_{\phi} F\right\|_{L^{p_{1} p_{2} q_{1} q_{2}}} \\
& \quad=\left(\int\left(\int\left(\int\left(\int\left|\left(\widetilde{V}_{\psi} F\right)(x, t, \xi, \nu)\right|^{p_{1}} d x\right)^{p_{2} / p_{1}} d t\right)^{q_{1} / p_{2}} d \xi\right)^{q_{2} / q_{1}} d \nu\right)^{1 / q_{2}} \\
& \quad<\infty
\end{aligned}
$$

with usual adjustments if $p_{1}=\infty, p_{2}=\infty, q_{1}=\infty$, and $/$ or $q_{2}=\infty$.

Note that the order of the list of variables in (1.2) is crucial as it indicates the order of integration in (1.3). We choose to list first the time variable $x$ followed by the time-shift variable $t$. The time variables are followed by the frequency variable $\xi$ and the frequency-shift variable $\nu$. Alternative orders of integration were considered, for example, in [2, $5,27,28]$.

Below, $\mathcal{L}(X, Y)$ denotes the space of all bounded linear operators mapping the Banach space $X$ to the Banach space $Y ; \mathcal{L}(X, Y)$ is equipped with the operator norm. Below, the conjugate exponent of $p \in[1, \infty]$ is denoted by $p^{\prime}$. Our main result follows.

Theorem 1.3. Let $p_{1}, p_{2}, p_{3}, p_{4}, q_{1}, q_{2}, q_{3}, q_{4} \in[1, \infty]$. Then there exists $C>0$ such that

$$
\left\|T_{\sigma}\right\|_{\mathcal{L}\left(M^{p_{1} q_{1}}, M^{p_{2} q_{2}}\right)} \leq C\|\sigma\|_{\widetilde{M}^{p_{3} p_{4} q_{3} q_{4}}}, \quad \sigma \in \widetilde{M}^{p_{3} p_{4} q_{3} q_{4}}\left(\mathbb{R}^{2 d}\right),
$$

if and only if

$$
\begin{aligned}
\frac{1}{p_{1}^{\prime}}+\frac{1}{p_{2}} & \leq \frac{1}{p_{3}}+\frac{1}{p_{4}}, p_{4} \leq \min \left\{p_{1}^{\prime}, p_{2}\right\}, \\
\frac{1}{q_{1}^{\prime}}+\frac{1}{q_{2}} & \leq \frac{1}{q_{3}}+\frac{1}{q_{4}}, q_{4} \leq \min \left\{q_{1}^{\prime}, q_{2}\right\} .
\end{aligned}
$$

Theorem 4.1 below is a variant of Theorem 1.3 that involves symbols in weighted modulation spaces.

Observe that (1.5) depends only on the parameters $p_{i}$, while (1.6) depends analogously only on the parameters $q_{i}$. That is, the conditions on decay in time and on decay in frequency, or, equivalently, on smoothness in frequency and on smoothness in time, on the Kohn-Nirenberg symbol are linked to the respective conditions on domain and range of the operator, but time and frequency remain independent of one another. See Figure 1 for an illustration of conditions (1.5) and (1.6).

An $L^{p}$-boundedness result for the introduced classes of pseudo-differential operators follows.

Corollary 1.4. Let $p, p_{3}, p_{4}, q, q_{3}, q_{4} \in[1, \infty]$. Assume

$$
\frac{1}{p^{\prime}}+\frac{1}{q} \leq \frac{1}{p_{3}}+\frac{1}{p_{4}}, \quad p_{4} \leq \min \left\{p^{\prime}, q\right\}
$$

and

$$
\begin{cases}\frac{1}{p}+\frac{1}{q} \leq \frac{1}{q_{3}}+\frac{1}{q_{4}}, q_{4} \leq \min \{p, q\}, & \text { if } p, q \in[1,2], \\ \frac{1}{p}+\frac{1}{q^{\prime}} \leq \frac{1}{q_{3}}+\frac{1}{q_{4}}, q_{4} \leq \min \left\{p, q^{\prime}\right\}, & \text { if } 1 \leq p \leq 2 \leq q, \\ \frac{1}{p^{\prime}}+\frac{1}{q^{\prime}} \leq \frac{1}{q_{3}}+\frac{1}{q_{4}}, q_{4} \leq \min \left\{p^{\prime}, q^{\prime}\right\}, & \text { if } 2 \leq \min \{p, q\}, \\ \frac{1}{p^{\prime}}+\frac{1}{q} \leq \frac{1}{q_{3}}+\frac{1}{q_{4}}, q_{4} \leq \min \left\{p^{\prime}, q\right\}, & \text { if } 1 \leq q \leq 2 \leq p .\end{cases}
$$

Then $T_{\sigma}: L^{p}\left(\mathbb{R}^{d}\right) \rightarrow L^{q}\left(\mathbb{R}^{d}\right)$ is bounded and there exists a constant $C>0$ such that

$$
\left\|T_{\sigma}\right\|_{\mathcal{L}\left(L^{p}, L^{q}\right)} \leq C\|\sigma\|_{\widetilde{M}^{p_{3} p_{4} q_{3} q_{4}}}, \quad \sigma \in \widetilde{M}^{p_{3} p_{4} q_{3} q_{4}}\left(\mathbb{R}^{2 d}\right) .
$$



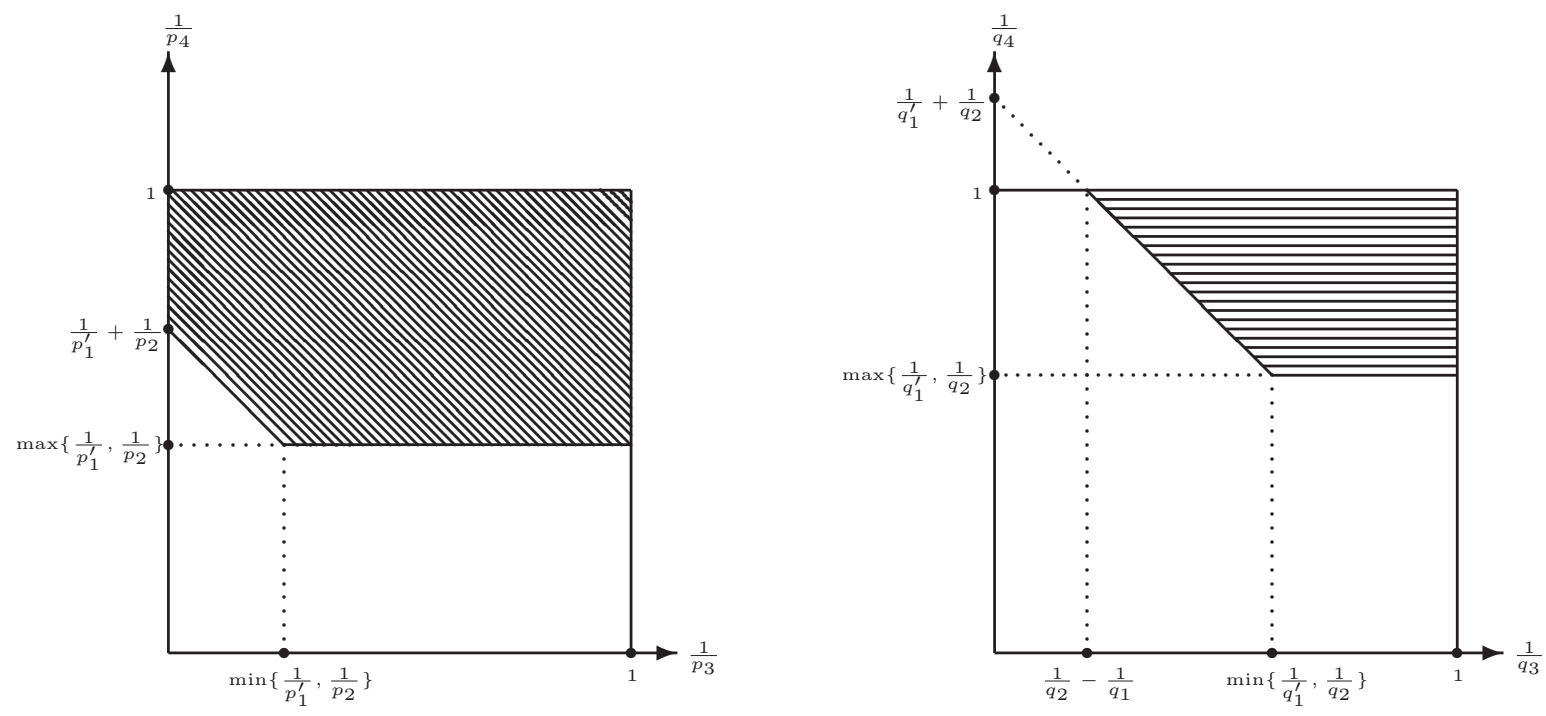

FIGURE 1. For fixed $p_{1}, p_{2}$ and $q_{1}, q_{2}$, we mark the regions of $\left(\frac{1}{p_{3}}, \frac{1}{p_{4}}\right)$ and $\left(\frac{1}{q_{3}}, \frac{1}{q_{4}}\right)$ for which every $\sigma \in \widetilde{M}^{p_{3} p_{4} q_{3} q_{4}}\left(\mathbb{R}^{2 d}\right)$ induces a bounded operator $T_{\sigma}: M^{p_{1} q_{1}}\left(\mathbb{R}^{d}\right) \rightarrow$ $M^{p_{2} q_{2}}\left(\mathbb{R}^{d}\right)$. In fact, for $\left(\frac{1}{p_{3}}, \frac{1}{p_{4}}\right)$ and $\left(\frac{1}{q_{3}}, \frac{1}{q_{4}}\right)$ in the hashed region, there exists $C>0$ with $\left\|T_{\sigma}\right\|_{\mathcal{L}\left(M^{p_{1} q_{1}}, M^{p_{2} q_{2}}\right)} \leq C\|\sigma\|_{\widetilde{M}^{p_{3} p_{4} q_{3} q_{4}}}$. The conditions on the time decay parameters $p_{1}, p_{2}, p_{3}, p_{4}$ are independent of the conditions on the frequency decay parameters $q_{1}, q_{2}, q_{3}, q_{4}$.

Corollary 1.4 encompasses, for example, the space of Hilbert-Schmidt operators on $L^{2}\left(\mathbb{R}^{d}\right)$, namely

$$
H S\left(L^{2}\left(\mathbb{R}^{d}\right)\right)=\left\{T_{\sigma}: \sigma \in \widetilde{M}^{2222}\left(\mathbb{R}^{2 d}\right)=L^{2}\left(\mathbb{R}^{2 d}\right)\right\} \subset \mathcal{L}\left(L^{2}\left(\mathbb{R}^{d}\right), L^{2}\left(\mathbb{R}^{d}\right)\right) .
$$

Moreover, Corollary 1.4 reconfirms $L^{2}$-boundedness of Sjöstrand class operators [25, 26],

$$
S j \subset\left\{T_{\sigma}: \sigma \in \widetilde{M}^{\infty 1 \infty 1}\left(\mathbb{R}^{2 d}\right)\right\} \subset \mathcal{L}\left(L^{2}\left(\mathbb{R}^{d}\right), L^{2}\left(\mathbb{R}^{d}\right)\right) .
$$

Using the weighted version of Theorem 1.3, namely, Theorem 4.1, we get the following boundedness result for Sobolev spaces.

Corollary 1.5. Let $p_{1}, p_{2}, p_{3}, p_{4} \in[1, \infty]$ and $s \in \mathbb{R}$. Let $w$ be a moderate weight function on $\mathbb{R}^{4 d}$ satisfying

$$
w(x, t, \nu, \xi) \leq\left(1+|\xi|^{2}\right)^{s / 2}\left(1+|\nu+\xi|^{2}\right)^{s / 2}, \quad x, t, \nu, \xi \in \mathbb{R}^{d} .
$$

Assume that

$$
\frac{1}{p_{1}^{\prime}}+\frac{1}{p_{2}} \leq \frac{1}{p_{3}}+\frac{1}{p_{4}}, \quad p_{4} \leq \min \left\{p_{1}^{\prime}, p_{2}\right\}
$$

Then

$$
\left\|T_{\sigma}\right\|_{\mathcal{L}\left(H_{s}^{p_{1}}, H_{s}^{p_{2}}\right)} \leq C\|\sigma\|_{\widetilde{M}_{w}^{p_{3} p_{4} 11}}, \quad \sigma \in \widetilde{M}_{w}^{p_{3} p_{4} 11}\left(\mathbb{R}^{2 d}\right)
$$

for some constant $C>0$.

The paper is structured as follows. Section 2 discusses mixed norm spaces and modulation spaces over Euclidean and over phase space in some detail. In Section 3, our boundedness results for pseudo-differential operators with symbols in modulation spaces over phase space are compared to results in the literature. Finally, in Section 4 we prove our main results, Theorem 1.3, Corollary 1.4, and Theorem 4.1. 


\section{Background on modulation spaces}

In the following, $x, \xi, t, \nu$ denote $d$-dimensional Euclidean variables. If not indicated differently, integration is with respect to the Lebesgue measure on $\mathbb{R}^{d}$.

Let $r=\left(r_{1}, r_{2}, \ldots, r_{n}\right)$ where $1 \leq r_{i}<\infty, i=1,2, \ldots, n$. The mixed norm space $L^{r}\left(\mathbb{R}^{n d}\right)$ is the set of all measurable functions $F$ on $\mathbb{R}^{n d}$ for which

$$
\begin{aligned}
& \|F\|_{L^{r}}= \\
& \qquad\left(\int_{\mathbb{R}} \ldots\left(\int_{\mathbb{R}}\left(\int_{\mathbb{R}}\left|F\left(x_{1}, \ldots, x_{n}\right)\right|^{r_{1}} d x_{1}\right)^{r_{2} / r_{1}} d x_{2} \ldots\right)^{r_{n} / r_{n-1}} d x_{n}\right)^{1 / r_{n}}
\end{aligned}
$$

is finite [1]. $L^{r}\left(\mathbb{R}^{n d}\right)$ is a Banach space with norm $\|\cdot\|_{L^{r}}$. Similarly, we define $L^{r}\left(\mathbb{R}^{n d}\right)$ where $r_{i}=\infty$ for some indices $i$.

If $n=2, r_{1}=p$ and $r_{2}=q$, then we denote $L^{r}\left(\mathbb{R}^{2 d}\right)$ by $L^{p q}\left(\mathbb{R}^{2 d}\right)$. Similarly, if $n=4$ and $r_{1}=p_{1}$, $r_{2}=p_{2}, r_{3}=p_{3}$ and $r_{4}=p_{4}$, we write $L^{p_{1} p_{2} p_{3} p_{4}}\left(\mathbb{R}^{4 d}\right)=L^{r}\left(\mathbb{R}^{4 d}\right)$.

Let $w$ be a nonnegative measurable function on $\mathbb{R}^{n d}$. We define $L_{w}^{r}\left(\mathbb{R}^{n d}\right)$ to be the space all $f$ on $\mathbb{R}^{n d}$ for which $w f$ is in $L^{r}\left(\mathbb{R}^{n d}\right)$. $L_{w}^{r}\left(\mathbb{R}^{n d}\right)$ is a Banach space with norm given by

$$
\|f\|_{L_{w}^{r}}=\|w f\|_{L^{r}} .
$$

In time-frequency analysis, it is advantageous to consider moderate weight functions $w$. To define these, let $\mathbb{R}_{0}^{+}$be the set of all nonnegative points in $\mathbb{R}$. Any locally integrable function $v: \mathbb{R}^{n d} \rightarrow \mathbb{R}_{0}^{+}$ with

$$
v(x+y) \leq v(x) v(y)
$$

is called submultiplicative. Moreover, if $w: \mathbb{R}^{n d} \rightarrow \mathbb{R}_{0}^{+}$is locally integrable with

$$
w(x+y) \leq C w(x) v(y)
$$

$C>0$, and $v$ submultiplicative, then $w$ is called moderate.

The short-time Fourier transform of a tempered distribution $f \in \mathcal{S}^{\prime}\left(\mathbb{R}^{n d}\right)$ with respect to the window $\psi \in \mathcal{S}\left(\mathbb{R}^{n d}\right)$ is given by

$$
V_{\psi} f(x, \xi)=\mathcal{F}\left(f T_{x} \bar{\psi}\right)(\xi)=\left(f, M_{\xi} T_{x} \psi\right)
$$

where $M_{\xi}$ and $T_{x}$ denote modulation and translation as defined above.

With $\phi(x)=e^{-\pi\|x\|^{2} / 2}, w$ moderate on $\mathbb{R}^{2 d}$, and $p, q \in[1, \infty]$, the modulation space $M_{w}^{p q}\left(\mathbb{R}^{d}\right)$ is the set of all tempered distributions $f \in \mathcal{S}^{\prime}\left(\mathbb{R}^{d}\right)$ such that

$$
V_{\phi} f \in L_{w}^{p q}\left(\mathbb{R}^{2 d}\right) .
$$

with respective Banach space norm. Clearly, if $w \equiv 1$, then $M_{w}^{p q}\left(\mathbb{R}^{d}\right)=M^{p q}\left(\mathbb{R}^{d}\right)$. Moreover, for any $s \in \mathbb{R}$ let

$$
w_{s}(x, \xi)=\left(1+|\xi|^{2}\right)^{s / 2}
$$

and denote $M_{w_{s}}^{p q}\left(\mathbb{R}^{d}\right)$ by $M_{s}^{p q}\left(\mathbb{R}^{d}\right)$.

Note that replacing the Gaussian function $\phi$ in the definition of modulation spaces by any other $\psi \in \mathcal{S}\left(\mathbb{R}^{d}\right) \backslash\{0\}$ defines the same space and an equivalent norm, a fact that will be used extensively below.

Recall that the Sobolev space $H_{s}^{p}\left(\mathbb{R}^{d}\right)$ consist of all tempered distributions $u \in \mathcal{S}^{\prime}\left(\mathbb{R}^{d}\right)$ for which $\|u\|_{H_{s}^{p}}=\left\|T_{w_{s}} u\right\|_{L^{p}}<\infty[27]$. For any $s \in \mathbb{R}$ and $1 \leq q \leq p \leq r \leq q^{\prime} \leq \infty$ we have

$$
M_{s}^{p q}\left(\mathbb{R}^{d}\right) \subseteq H_{s}^{r}\left(\mathbb{R}^{d}\right),
$$

and for some $C>0$,

$$
\|f\|_{H_{s}^{r}} \leq C\|f\|_{M_{s}^{p q}}, \quad f \in M_{s}^{p q}\left(\mathbb{R}^{d}\right) .
$$


Similarly, $1 \leq q^{\prime} \leq r \leq p \leq q \leq \infty$ implies

$$
H_{s}^{r}\left(\mathbb{R}^{d}\right) \subseteq M_{s}^{p q}\left(\mathbb{R}^{d}\right)
$$

and for some constant $C>0$,

$$
\|f\|_{M_{s}^{p q}} \leq C\|f\|_{H_{s}^{r}}, \quad f \in H_{s}^{r}\left(\mathbb{R}^{d}\right) .
$$

Let $\mathcal{F} L^{p}\left(\mathbb{R}^{d}\right)$ be the space of all tempered distributions $f$ in $\mathcal{S}^{\prime}\left(\mathbb{R}^{d}\right)$ for which there exists a function $h \in L^{p}\left(\mathbb{R}^{d}\right)$ such that $\hat{h}=f$. Then $\mathcal{F} L^{p}\left(\mathbb{R}^{d}\right)$ is a Banach space equipped with the norm

$$
\|f\|_{\mathcal{F} L^{p}}=\|h\|_{L^{p}} .
$$

The following proposition shows that modulation space norms of compactly supported or bandlimited functions can be estimated using $\mathcal{F} L^{p}$ and $L^{p}$ norms, respectively $[6,8,22,29]$.

Proposition 2.1. For $K \subset \mathbb{R}^{d}$ compact and $p, q \in[1, \infty]$ there are constants $A, B, C, D>0$ with

(i) $\quad A\|f\|_{\mathcal{F} L^{q}} \leq\|f\|_{M^{p q}} \leq B\|f\|_{\mathcal{F} L^{q}}, \quad f \in \mathcal{S}^{\prime}\left(\mathbb{R}^{d}\right)$ with supp $f \subseteq K$;

(ii) $\quad C\|f\|_{L^{p}} \leq\|f\|_{M^{p q}} \leq D\|f\|_{L^{p}}, \quad f \in \mathcal{S}^{\prime}\left(\mathbb{R}^{d}\right)$ with supp $\widehat{f} \subseteq K$.

In the following, we shall denote norm equivalences as in statement $(i)$ above by

$$
\|f\|_{\mathcal{F} L^{q}} \asymp\|f\|_{M^{p q}}, \quad f \in \mathcal{S}^{\prime}\left(\mathbb{R}^{d}\right), \quad \operatorname{supp} f \subseteq K .
$$

Similarly, statement $(i i)$ becomes

$$
\|f\|_{L^{p}} \asymp\|f\|_{M^{p q}}, \quad f \in \mathcal{S}^{\prime}\left(\mathbb{R}^{d}\right), \quad \operatorname{supp} \widehat{f} \subseteq K .
$$

The symplectic Fourier transform of $F \in \mathcal{S}\left(\mathbb{R}^{2 d}\right)$ given in (1.1) is a $2 d$-dimensional Fourier transform followed by a rotation of phase space by $\frac{\pi}{2}$. This implies that the symplectic Fourier transform shares most properties with the Fourier transform, for example, Proposition 2.1 remains true when replacing the Fourier transform by the symplectic Fourier transform.

Let $p_{1}, p_{2}, q_{1}, q_{2} \in[1, \infty]$ and let $w$ be a $v$-moderate weight function on $\mathbb{R}^{4 d}$. The weighted modulation space over phase space $\widetilde{M}_{w}^{p_{1} p_{2} q_{1} q_{2}}\left(\mathbb{R}^{2 d}\right)$ is the set of all tempered distributions $F \in \mathcal{S}^{\prime}\left(\mathbb{R}^{2 d}\right)$ for which $\widetilde{V}_{\psi} F \in L_{w}^{p_{1} p_{2} q_{1} q_{2}}\left(\mathbb{R}^{4 d}\right)$.

Recapitulate that for $F \in \mathcal{S}^{\prime}\left(\mathbb{R}^{2 d}\right)$, we have $\widetilde{V}_{\psi} F(x, t, \xi, \nu)=V_{\psi} F(x, \xi, \nu,-t)$,

$$
\begin{aligned}
& \|F\|_{\widetilde{M}^{p_{1} p_{2} q_{1} q_{2}}}=\left\|\widetilde{V}_{\phi} F\right\|_{L^{p_{1} p_{2} q_{1} q_{2}}} \\
& \quad=\left(\int\left(\int\left(\int\left(\int\left|\widetilde{V}_{\psi} F(x, t, \xi, \nu)\right|^{p_{1}} d x\right)^{p_{2} / p_{1}} d t\right)^{q_{1} / p_{2}} d \xi\right)^{q_{2} / q_{1}} d \nu\right)^{1 / q_{1}},
\end{aligned}
$$

and

$$
\begin{aligned}
& \|F\|_{M^{p_{1} q_{1} q_{2} p_{2}}}=\left\|V_{\phi} F\right\|_{L^{p_{1} q_{1} q_{2} p_{2}}} \\
& \quad=\left(\int\left(\int\left(\int\left(\int\left|V_{\psi} F(x, \xi, \nu, t)\right|^{p_{1}} d x\right)^{q_{1} / p_{1}} d \xi\right)^{q_{2} / q_{1}} d \nu\right)^{p_{1} / q_{1}} d t\right)^{1 / p_{1}},
\end{aligned}
$$

with usual adjustments if $p_{1}=\infty, p_{2}=\infty, q_{1}=\infty$, and/or $q_{2}=\infty$. This shows that the definition of $\widetilde{M}^{p_{1}, p_{2}, q_{1}, q_{4}}\left(\mathbb{R}^{2 d}\right)$ is based on changing the order of integration and on relabeling the integration exponents accordingly. Mixed $L^{p}$ spaces are sensitive on the order of integration, and, hence, $\widetilde{M}^{p_{1} p_{2} q_{1} q_{2}}\left(\mathbb{R}^{2 d}\right) \nsubseteq$ $M^{p_{1} p_{2} q_{1} q_{2}}\left(\mathbb{R}^{2 d}\right)$ and $M^{p_{1} p_{2} q_{1} q_{2}}\left(\mathbb{R}^{2 d}\right) \nsubseteq \widetilde{M}^{p_{1} p_{2} q_{1} q_{2}}\left(\mathbb{R}^{2 d}\right)$ in general. But for $1 \leq p \leq q \leq \infty$, Minkowski's inequality

$$
\left(\int\left(\int|F(x, y)|^{p} d x\right)^{q / p} d y\right)^{p} \leq\left(\int\left(\int|F(x, y)|^{q} d y\right)^{p / q} d x\right)^{q}
$$

(with adjustments for $p=\infty$ and/or $q=\infty$ ) holds and implies the following. 
Proposition 2.2. Let $p_{1}, p_{2}, q_{1}, q_{2} \in[1, \infty]$ and $w$ be a moderate weight function on $\mathbb{R}^{4 d}$.

(i) If $p_{2} \leq \min \left\{q_{1}, q_{2}\right\}$, then $M_{w}^{p_{1} q_{1} q_{2} p_{2}}\left(\mathbb{R}^{2 d}\right) \subseteq \widetilde{M}_{w}^{p_{1} p_{2} q_{1} q_{2}}\left(\mathbb{R}^{2 d}\right)$ and $\|\sigma\|_{\widetilde{M}_{w}^{p_{1} p_{2} q_{1} q_{2}}} \leq\|\sigma\|_{M_{w}^{p_{1} q_{1} q_{2} p_{2}}}$.

(ii) If $\max \left\{q_{1}, q_{2}\right\} \leq p_{2}$, then $\widetilde{M}_{w}^{p_{1} p_{2} q_{1} q_{2}}\left(\mathbb{R}^{2 d}\right) \subseteq M_{w}^{p_{1} q_{1} q_{2} p_{2}}\left(\mathbb{R}^{2 d}\right)$ and

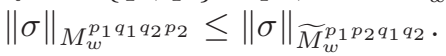

Note that results similar to ours could also be achieved using symbols in $M_{w}^{p_{3} p_{4} q_{3} q_{4}}\left(\mathbb{R}^{2 d}\right)$, but the so obtained results would be weaker and they would necessitate the additional condition $p_{4} \leq \min \left\{q_{3}, q_{4}\right\}$.

Modulation spaces over phase space share most of the properties of ordinary modulation spaces. For example, if $p_{1} \leq \widetilde{p}_{1}, p_{2} \leq \widetilde{p}_{2}, q_{1} \leq \widetilde{q}_{1}$, and $q_{2} \leq \widetilde{q}_{2}$, then

$$
\widetilde{M}_{w}^{p_{1} p_{2} q_{1} q_{2}}\left(\mathbb{R}^{2 d}\right) \subseteq \widetilde{M}_{w}^{\widetilde{p}_{1} \widetilde{p}_{2} \widetilde{q}_{1} \widetilde{q}_{2}}\left(\mathbb{R}^{2 d}\right),
$$

and

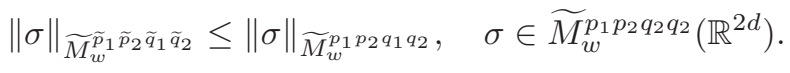

Furthermore, for $p_{1}, p_{2}, q_{1}, q_{2} \in[1, \infty]$, the dual of $\widetilde{M}_{w}^{p_{1} p_{2} q_{1} q_{2}}\left(\mathbb{R}^{2 d}\right)$ is $\widetilde{M}_{w}^{p_{1}^{\prime} p_{2}^{\prime} q_{1}^{\prime} q_{2}^{\prime}}\left(\mathbb{R}^{2 d}\right)$ where $p_{1}^{\prime}, p_{2}^{\prime}, q_{1}^{\prime}, q_{2}^{\prime}$ are conjugate exponents of $p_{1}, p_{2}, q_{1}, q_{2}$.

The proofs of such results for modulation spaces over phase space are similar to the ones for the ordinary modulation spaces [14] and are omitted.

\section{Comparison of Theorem 1.3 to results in the literature}

Cordero and Nicola as well as Toft proved the following theorem on $M^{p q}$-boundedness for classes of pseudo-differential operators with symbols in $M^{s_{1} s_{1} s_{2} s_{2}}\left(\mathbb{R}^{2 d}\right)$, see Theorem 5.2 in [5] and Theorem 4.3 in [27].

Theorem 3.1. Let $p, q, s_{1}, s_{2} \in[1, \infty]$. Then for some $C>0$,

$$
\left\|T_{\sigma}\right\|_{\mathcal{L}\left(M^{p q}, M^{p q}\right)} \leq C\|\sigma\|_{M^{s_{1} s_{1} s_{2} s_{2}}}, \quad \sigma \in M^{s_{1} s_{1} s_{2} s_{2}}\left(\mathbb{R}^{2 d}\right),
$$

if and only if

$$
s_{2} \leq \min \left\{p, p^{\prime}, q, q^{\prime}, s_{1}^{\prime}\right\} .
$$

Roughly speaking, to employ Theorem 3.1, we need to ensure that $\sigma(x, \xi)$ has $L^{s}$ 'decay' in $x$ and $\xi$ and that $\mathcal{F} \sigma(\nu,-t)=\mathcal{F}_{s} \sigma(t, \nu)$ has $L^{\min \left\{p, p^{\prime}, q, q^{\prime}, s^{\prime}\right\}}$ 'decay' in $t$ and $\nu$. To apply Theorem 1.3, it suffices to ensure that $\sigma(x, \xi)$ has $L^{s_{1}}$ 'decay' in $x$ and $L^{s_{2}}$ 'decay' in $\xi$, and that $\mathcal{F}_{s} \sigma(t, \nu)$ has $L^{\min \left\{p, p^{\prime}, s_{1}^{\prime}\right\}}$ 'decay' in $t$ and $L^{\min \left\{q, q^{\prime}, s_{2}\right\}}$ 'decay' in $\nu$.

Using embeddings such as (2.3), we observe that indeed Theorem 3.1 provides boundedness of $T_{\sigma}$ if

$$
\sigma \in \bigcup_{s=\max \left\{p, p^{\prime}, q, q^{\prime}\right\}}^{\infty} M^{s s s^{\prime} s^{\prime}} \subseteq \bigcup_{s=\max \left\{p, p^{\prime}, q, q^{\prime}\right\}}^{\infty} \widetilde{M}^{s s^{\prime} s s^{\prime}}
$$

while Theorem 1.3 provides boundedness of $T_{\sigma}$ if

$$
\sigma \in \bigcup_{s_{1}=\max \left\{p, p^{\prime}\right\}}^{\infty} \bigcup_{s_{2}=\max \left\{q, q^{\prime}\right\}}^{\infty} \widetilde{M}^{s_{1} s_{1}^{\prime} s_{2} s_{2}^{\prime}} .
$$

The set inclusion in (3.2) is based on Theorem 2.2 and the fact that $s \geq \max \left\{p, p^{\prime}\right\}$ implies $s \geq 2 \geq s^{\prime}$.

As $L^{2}=M^{22}$, Theorem 3.1 implies the following $L^{2}$-boundedness result. 
Corollary 3.2. Let $r, s \in[1, \infty]$. Then for some $C>0$,

$$
\left\|T_{\sigma}\right\|_{\mathcal{L}\left(L^{2}, L^{2}\right)} \leq C\|\sigma\|_{M^{r r s s}}, \quad \sigma \in M^{r r s s}\left(\mathbb{R}^{2 d}\right),
$$

if and only if

$$
s \leq \min \left\{2, r^{\prime}\right\} .
$$

Corollary 3.2 has been obtained earlier in 2003 by Gröchenig and Heil [15]. As comparison, we formulate a similar consequence of Theorem 1.3.

Corollary 3.3. For $r, s \in[2, \infty]$, there exists a constant $C>0$ such that

$$
\left\|T_{\sigma}\right\|_{\mathcal{L}\left(L^{2}, L^{2}\right)} \leq C\|\sigma\|_{\widetilde{M}^{r r^{\prime} s s^{\prime}}}, \quad \sigma \in \widetilde{M}^{r r^{\prime} s s^{\prime}}\left(\mathbb{R}^{2 d}\right) .
$$

Note that Theorem 3.1 does not imply that $T_{\sigma}: L^{2}\left(\mathbb{R}^{d}\right) \rightarrow L^{2}\left(\mathbb{R}^{d}\right)$ is bounded for $\sigma \in M^{\infty 221}\left(\mathbb{R}^{2 d}\right)$. But as $M^{\infty 221}\left(\mathbb{R}^{2 d}\right) \subseteq \widetilde{M}^{\infty 122}\left(\mathbb{R}^{2 d}\right)$, Theorem 1.3 indeed implies $L^{2}$-boundedness in this case.

Theorem 1.3 implies the following result for compositions of product and convolution operators.

Corollary 3.4. For $p, q \in[2, \infty]$, let $h_{1} \in M^{p q^{\prime}}\left(\mathbb{R}^{d}\right)$ and $h_{2} \in M^{p^{\prime} q}\left(\mathbb{R}^{d}\right)$. Define

$$
T f=h_{1} \cdot\left(h_{2} * f\right), \quad f \in L^{2}\left(\mathbb{R}^{d}\right),
$$

and

$$
H f=\left(h_{1} \cdot f\right) * h_{2}, \quad f \in L^{2}(\mathbb{R}) .
$$

Then $T$ and $H$ are $L^{2}$-bounded operators and there exists a positive constant $C$ such that

$$
\|T\|_{\mathcal{L}\left(L^{2}, L^{2}\right)} \leq C\left\|h_{1}\right\|_{M^{p q^{\prime}}}\left\|h_{2}\right\|_{M^{p^{\prime} q}},
$$

and

$$
\|H\|_{\mathcal{L}\left(L^{2}, L^{2}\right)} \leq C\left\|h_{1}\right\|_{M^{p q^{\prime}}}\left\|h_{2}\right\|_{M^{p^{\prime} q}} .
$$

The proof of Corollary 3.4 follows immediately from Corollary 3.3, Lemma 4.9 and Lemma 4.10.

\section{Proof of Theorem 1.3, Corollary 1.4, and Theorem 4.1}

\subsection{Proof of Theorem 4.1 and thereby of (1.5) and (1.6) implies (1.4) in Theorem 1.3}

In this section we prove the weighted version of one implication of Theorem 1.3.

Theorem 4.1. Let $w_{1}, w_{2}$ be moderate weight functions on $\mathbb{R}^{2 d}$ and $w$ be a moderate weight function on $\mathbb{R}^{4 d}$ that satisfy

$$
w_{2}(x, \nu+\xi) \leq w(x, t, \nu, \xi) w_{1}(x-t, \xi) .
$$

Let $p_{1}, p_{2}, p_{3}, p_{4}, q_{1}, q_{2}, q_{3}, q_{4} \in[1, \infty]$ be such that

$$
\begin{aligned}
& \frac{1}{p_{1}^{\prime}}+\frac{1}{p_{2}} \leq \frac{1}{p_{3}}+\frac{1}{p_{4}}, p_{4} \leq \min \left\{p_{1}^{\prime}, p_{2}\right\}, \\
& \frac{1}{q_{1}^{\prime}}+\frac{1}{q_{2}} \leq \frac{1}{q_{3}}+\frac{1}{q_{4}}, q_{4} \leq \min \left\{q_{1}^{\prime}, q_{2}\right\} .
\end{aligned}
$$

Then there exists a constant $C>0$ such that

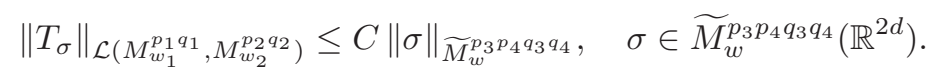


To prove Theorem 4.1 we need some preparation. For functions $f$ and $g$ in $\mathcal{S}\left(\mathbb{R}^{d}\right)$, the Rihaczek transform $R(f, g)$ of $f$ and $g$ is defined by

$$
R(f, g)(x, \xi)=e^{2 \pi i x \cdot \xi} \hat{f}(\xi) \overline{g(x)} .
$$

Pseudo-differential operators are related to Rihaczek transforms by

$$
\left(T_{\sigma} f, g\right)=(\sigma, \overline{R(f, g)})
$$

for $f$ and $g$ in $\mathcal{S}\left(\mathbb{R}^{d}\right)$. We define $A, T_{A}$ by

$$
\left(T_{A} F\right)(x, t)=F(A(x, t))=F(x-t, x) .
$$

Then

$$
\overline{R(f, g)}(x, \xi)=\mathcal{F}_{t \rightarrow \xi}\left(T_{A}(\bar{f} \otimes g)(x, \cdot)\right) .
$$

Lemma 4.2. Let $\varphi$ be a real valued Schwartz function on $\mathbb{R}^{d}$. Then for all $f$ and $g$ in $\mathcal{S}\left(\mathbb{R}^{d}\right)$

$$
V_{T_{A}(\varphi \otimes \varphi)} T_{A}(\bar{f} \otimes g)(x, t, \nu, \xi)=\overline{V_{\varphi} f(x-t, \xi)} V_{\varphi} g(x, \nu+\xi), \quad f, g \in \mathcal{S}\left(\mathbb{R}^{d}\right) .
$$

Proof. We compute

$$
\begin{aligned}
& V_{T_{A}(\varphi \otimes \varphi)} T_{A}(\bar{f} \otimes g)(x, t, \nu, \xi) \\
& =\iint e^{-2 \pi i(\widetilde{x} \nu+\widetilde{t \xi})} T_{A}(\bar{f} \otimes g)(\widetilde{x}, \widetilde{t}) T_{A}(\varphi \otimes \varphi)(\widetilde{x}-x, \widetilde{t}-t) d \widetilde{x} d \widetilde{t} \\
& =\int\left(\int e^{-2 \pi i \widetilde{t} \xi} \bar{f}(\widetilde{x}-\widetilde{t}) \varphi(\widetilde{x}-x-\widetilde{t}+t) d \widetilde{t}\right) e^{-2 \pi i \widetilde{x} \nu} g(\widetilde{x}) \varphi(\widetilde{x}-x) d \widetilde{x} \\
& =\iint \bar{f}(s) g(\widetilde{x}) e^{-2 \pi i \nu \widetilde{x}-2 \pi i \xi(\widetilde{x}-s)} \varphi(s-(x-t)) \varphi(\widetilde{x}-x) d \widetilde{x} d s \\
& =\left(\overline{\int e^{-2 \pi i \xi s} f(s) \varphi(s-(x-t)) d s}\right)\left(\int e^{-2 \pi i(\nu+\xi) \widetilde{x}} g(\widetilde{x}) \varphi(\widetilde{x}-x) d \widetilde{x}\right) \\
& =\overline{V_{\varphi} f(x-t, \xi)} V_{\varphi} g(x, \nu+\xi) \text {. }
\end{aligned}
$$

Lemma 4.3. Let $\varphi \in \mathcal{S}\left(\mathbb{R}^{d}\right)$ be a nonzero even real valued Schwartz function on $\mathbb{R}^{d}$. Then

$$
V_{\overline{R(\varphi, \varphi)}} \overline{R(f, g)}(x, \xi, \nu, t)=e^{-2 \pi i \xi t} V_{T_{A}(\varphi \otimes \varphi)} T_{A}(\bar{f} \otimes g)(x,-t, \nu, \xi) \quad f, g \in \mathcal{S}\left(\mathbb{R}^{d}\right) .
$$

Proof. For $f$ and $g$ in $\mathcal{S}\left(\mathbb{R}^{d}\right)$

$$
\begin{aligned}
& V_{\overline{R(\varphi, \varphi)}} \overline{R(f, g)}(x, \xi, \nu, t) \\
& \quad=\iint e^{-2 \pi i(\nu \widetilde{x}+t \widetilde{\xi})} \overline{R(f, g)(\widetilde{x}, \widetilde{\xi})} R(\varphi, \varphi)(\widetilde{x}-x, \widetilde{\xi}-\xi) d \widetilde{x} d \widetilde{\xi} \\
& \quad=\iint e^{-2 \pi i(\nu \widetilde{x}+t \widetilde{\xi})} \mathcal{F}_{\widetilde{t} \rightarrow \widetilde{\xi}}(\bar{f}(\widetilde{x}-\cdot)) g(\widetilde{x}) \overline{\mathcal{F}_{\widetilde{t} \rightarrow \widetilde{\xi}-\xi}(\varphi(\widetilde{x}-x-\cdot))} \varphi(\widetilde{x}-x) d \widetilde{x} d \widetilde{\xi} \\
& \quad=\iint e^{-2 \pi i(\nu \widetilde{x}+t \widetilde{\xi})} \mathcal{F}_{\widetilde{t} \rightarrow \widetilde{\xi}}(\bar{f}(\widetilde{x}-\cdot)) g(\widetilde{x}) \mathcal{F}_{\widetilde{t} \rightarrow \xi-\widetilde{\xi}}(\varphi(\widetilde{x}-x-\cdot)) \varphi(\widetilde{x}-x) d \widetilde{x} d \widetilde{\xi}
\end{aligned}
$$


On the other hand, Parseval's identity gives

$$
\begin{aligned}
& V_{T_{A}(\varphi \otimes \varphi)} T_{A}(\bar{f} \otimes g)(x, t, \nu, \xi) \\
& =\iint e^{-2 \pi i(\widetilde{x} \nu+\widetilde{t} \xi)} T_{A}(\bar{f} \otimes g)(\widetilde{x}, \widetilde{t}) T_{A}(\varphi \otimes \varphi)(\widetilde{x}-x, \widetilde{t}-t) d \widetilde{x} d \widetilde{t} \\
& =\int\left(\int e^{-2 \pi i \widetilde{t} \xi} \bar{f}(\widetilde{x}-\widetilde{t}) \varphi(\widetilde{x}-x-\widetilde{t}+t) d \widetilde{t}\right) e^{-2 \pi i \widetilde{x} \nu} g(\widetilde{x}) \varphi(\widetilde{x}-x) d \widetilde{x} \\
& =\iint \mathcal{F}_{\widetilde{t} \rightarrow \widetilde{\xi}}(\bar{f}(\widetilde{x}-\cdot)) \mathcal{F}_{\widetilde{t} \rightarrow \widetilde{\xi}}^{-1}\left(e^{-2 \pi i \widetilde{t}} \varphi(\widetilde{x}-x+t-\cdot)\right) e^{-2 \pi i \widetilde{x} \nu} g(\widetilde{x}) \varphi(\widetilde{x}-x) d \widetilde{\xi} d \widetilde{x} .
\end{aligned}
$$

But

$$
\mathcal{F}_{\widetilde{t} \rightarrow \widetilde{\xi}}^{-1}\left(e^{-2 \pi i \widetilde{\xi}} \varphi(\widetilde{x}-x+t-\cdot)\right)=e^{-2 \pi i t(\xi-\widetilde{\xi})} \mathcal{F}_{\gamma \rightarrow \xi-\widetilde{\xi}}(\varphi(\widetilde{x}-x-\cdot))
$$

therefore,

$$
\begin{array}{r}
V_{T_{A}(\varphi \otimes \varphi)} T_{A}(\bar{f} \otimes g)(x, t, \nu, \xi)=e^{-2 \pi i t \xi} \iint e^{2 \pi i(t \widetilde{\xi}-v \widetilde{x})} \mathcal{F}_{\widetilde{t} \rightarrow \widetilde{\xi}}(\bar{f}(\widetilde{x}-\cdot)) \times \\
\mathcal{F}_{\widetilde{t} \rightarrow \xi-\widetilde{\xi}}(\varphi(\widetilde{x}-x-\cdot)) g(\widetilde{x}) \varphi(\widetilde{x}-x) d \widetilde{x} d \widetilde{\xi}
\end{array}
$$

Combining this identity with (4.2) completes the proof.

Proposition 4.4. Let $w_{1}, w_{2}, w$ be moderate functions that satisfy

$$
w(x, t, \nu, \xi) \leq w_{1}(x-t, \xi) w_{2}(x, \nu+\xi) .
$$

Let $\varphi$ be a nonzero real valued Schwartz function on $\mathbb{R}^{d}$ and define

$$
\mathcal{V}_{T_{A}(\varphi \otimes \varphi)} T_{A}(\bar{f} \otimes g)(x, t, \xi, \nu)=V_{T_{A}(\varphi \otimes \varphi)} T_{A}(\bar{f} \otimes g)(x, t, \nu, \xi), \quad f, g \in \mathcal{S}\left(\mathbb{R}^{d}\right) .
$$

If $p_{1}, p_{2}, p_{3}, p_{4}, q_{1}, q_{2}, q_{3}, q_{4} \in[1, \infty]$ satisfy

$$
\begin{aligned}
& \frac{1}{p_{1}}+\frac{1}{p_{2}}=\frac{1}{p_{3}}+\frac{1}{p_{4}}, p_{3} \leq \min \left\{p_{1}, p_{2}, p_{4}\right\}, \\
& \frac{1}{q_{1}}+\frac{1}{q_{2}}=\frac{1}{q_{3}}+\frac{1}{q_{4}}, q_{3} \leq \min \left\{q_{1}, q_{2}, q_{4}\right\},
\end{aligned}
$$

then

$$
\left\|\mathcal{V}_{T_{A}(\varphi \otimes \varphi)} T_{A}(\bar{f} \otimes g)\right\|_{L_{w}^{p_{3} p_{4} q_{3} q_{4}}} \leq\|f\|_{M_{w_{1}}^{p_{1} q_{1}}}\|g\|_{M_{w_{2}}^{p_{2} q_{2}}} .
$$

Proof. Lemma 4.2 implies

$$
\mathcal{V}_{T_{A}(\varphi \otimes \varphi)} T_{A}(\bar{f} \otimes g)(x, t, \xi, \nu)=\overline{V_{\varphi} f(x-t, \xi)} V_{\varphi} g(x, \nu+\xi) .
$$

So, by (4.1),

$$
\begin{aligned}
& \left\|w(\cdot, t, \xi, \nu) \mathcal{V}_{T_{A}(\varphi \otimes \varphi)} T_{A}(\bar{f} \otimes g)(\cdot, t, \xi, \nu)\right\|_{L^{p_{3}}} \\
& \quad \leq\left(\int\left|w_{1}(x-t, \xi)\left(V_{\varphi} f\right)(x-t, \xi)\right|^{p_{3}}\left|w_{2}(x, \nu+\xi)\left(V_{\varphi} g\right)(x, \nu+\xi)\right|^{p_{3}} d x\right)^{1 / p_{3}} \\
& \quad=\left(\left|w_{2}(\cdot, \nu+\xi) V_{\varphi} g(\cdot, \nu+\xi)\right|^{p_{3}} *\left|w_{1}(\cdot, \xi) V_{\varphi} f(\cdot, \xi)\right|^{p_{3}}(t)\right)^{1 / p_{3}} \cdot
\end{aligned}
$$

Then, (4.4) implies

$$
\frac{1}{r_{1}}+\frac{1}{s_{1}}=1+\frac{1}{a_{1}}
$$


with $r_{1}=p_{2} / p_{3} \geq 1, s_{1}=p_{1} / p_{3} \geq 1$ and $a_{1}=p_{4} / p_{3} \geq 1$. Hence, we can apply Young's inequality and obtain

$$
\begin{aligned}
& \left\|w(\cdot, \cdot, \xi, \nu) \mathcal{V}_{T_{A}(\varphi \otimes \varphi)} T_{A}(\bar{f} \otimes g)(\cdot, \cdot, \xi, \nu)\right\|_{L^{p_{3} p_{4}}} \\
& \quad=\left\|\left|w_{2}(\cdot, \nu+\xi) V_{\varphi} g(\cdot, \nu+\xi)\right|^{p_{3}} *\left|w_{1}(\cdot, \xi) V_{\varphi} f(\cdot, \xi)\right|^{p_{3}}\right\|_{L^{a_{1}}}^{1 / p_{3}} \\
& \quad \leq\left\|\left|w_{2}(\cdot, \nu+\xi) V_{\varphi} g(\cdot, \nu+\xi)\right|^{p_{3}}\right\|_{L^{r_{1}}}^{1 / p_{3}}\left\|\left|w_{1}(\cdot, \xi) V_{\varphi} f(\cdot, \xi)\right|^{p_{3}}\right\|_{L^{s_{1}}}^{1 / p_{3}} .
\end{aligned}
$$

To bound (4.5), we note the integral with respect to $\xi$ is again a convolution. In fact (4.4) leads to

$$
\frac{1}{r_{2}}+\frac{1}{s_{2}}=1+\frac{1}{a_{2}}
$$

where $r_{2}=q_{2} / q_{3}, s_{2}=q_{1} / q_{3}$ and $a_{2}=q_{4} / q_{3}$. Young's inequality then implies

$$
\begin{aligned}
&\left\|w \mathcal{V}_{T_{A}(\varphi \otimes \varphi)} T_{A}(\bar{f} \otimes g)\right\|_{L^{p_{3} p_{4} q_{3} q_{4}}} \\
& \leq\left(\int\left(\int\left|w_{2}(x, y) V_{\varphi} g(x, y)\right|^{p_{3} r_{1}} d x\right)^{\left(r_{2} q_{3}\right) /\left(p_{3} r_{1}\right)} d y\right)^{1 /\left(r_{2} q_{3}\right)} \times \\
& \quad\left(\int\left(\int\left|w_{1}(x, y) V_{\varphi} f(x, y)\right|^{p_{3} s_{1}} d x\right)^{\left(s_{2} q_{3}\right) /\left(p_{3} s_{1}\right)} d y\right)^{\left(1 / s_{2} q_{3}\right)} \\
&=\|f\|_{M_{w_{1}}^{p_{1} q_{1}}\|g\|_{M_{w_{2}}^{p_{2} q_{2}},},}
\end{aligned}
$$

which completes the proof.

Now, we are ready to give sufficient conditions on the boundedness of pseudo-differential operators with symbols in $\widetilde{M}^{p_{3} p_{4} q_{3} q_{4}}\left(\mathbb{R}^{2 d}\right)$.

Lemma 4.5. Let $w_{1}, w_{2}, w$ be moderate weight functions that satisfy (4.1). Let $p_{1}, p_{2}, p_{3}, p_{4}, q_{1}, q_{2}, q_{3}, q_{4} \in[1, \infty]$ be such that

$$
\begin{aligned}
& \frac{1}{p_{3}} \in\left[\frac{1}{p_{1}^{\prime}}+\frac{1}{p_{2}}-\frac{1}{p_{4}}, \min \left\{\frac{1}{p_{1}^{\prime}}, \frac{1}{p_{2}}, \frac{1}{p_{4}}\right\}\right], \\
& \frac{1}{q_{3}} \in\left[\frac{1}{q_{1}^{\prime}}+\frac{1}{q_{2}}-\frac{1}{q_{4}}, \min \left\{\frac{1}{q_{1}^{\prime}}, \frac{1}{q_{2}}, \frac{1}{q_{4}}\right\}\right] .
\end{aligned}
$$

Then there exists a constant $C>0$ such that

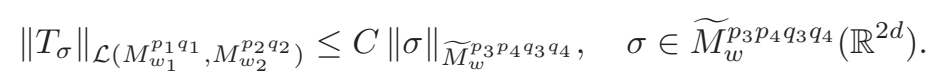

Proof. Let us first assume $p_{1}, p_{2}, p_{3}, p_{4}, q_{1}, q_{2}, q_{3}, q_{4} \in[1, \infty]$ satisfy (4.6) and in addition

$$
\frac{1}{p_{1}^{\prime}}+\frac{1}{p_{2}}=\frac{1}{p_{3}}+\frac{1}{p_{4}} \text { and } \quad \frac{1}{q_{1}^{\prime}}+\frac{1}{q_{2}}=\frac{1}{q_{3}}+\frac{1}{q_{4}}
$$

Let $f, g \in \mathcal{S}\left(\mathbb{R}^{d}\right)$. Since the dual of $\widetilde{M}_{w}^{p_{3} p_{4} q_{3} q_{4}}\left(\mathbb{R}^{2 d}\right)$ is $\widetilde{M}_{1 / w}^{p_{3}^{\prime} p_{4}^{\prime} q_{3}^{\prime} q_{4}^{\prime}}\left(\mathbb{R}^{2 d}\right)$, it follows that

$$
\begin{aligned}
\left|\left(T_{\sigma} f, g\right)\right| & =|(\sigma, \overline{R(f, g)})| \\
& \leq\|\sigma\|_{\widetilde{M}_{w}^{p_{3} p_{4} q_{3} q_{4}}}\|\overline{R(f, g)}\|_{\widetilde{M}_{1 / w}^{p_{3}^{\prime} p_{4}^{\prime} q_{3}^{\prime} q_{4}^{\prime}}} .
\end{aligned}
$$

To obtain (4.7), it is enough to show that there exists $C>0$ such that

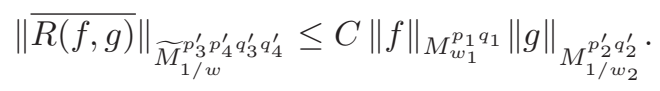


Let $\varphi$ be a nonzero real valued even function in $\mathcal{S}\left(\mathbb{R}^{d}\right)$. Then by Lemma 4.3 ,

$$
\begin{aligned}
\left|\mathcal{V}_{\overline{R(\varphi, \varphi)}} \overline{R(f, g)}(x, t, \xi, \nu)\right| & =\left|V_{\overline{R(\varphi, \varphi)}} \overline{R(f, g)}(x, \xi, \nu,-t)\right| \\
& =\left|V_{T_{A}(\varphi \otimes \varphi)} T_{A}(\bar{f} \otimes g)(x, t, \nu, \xi)\right| \\
& =\left|\mathcal{V}_{T_{A}(\varphi \otimes \varphi)} T_{A}(\bar{f} \otimes g)(x, t, \xi, \nu)\right| .
\end{aligned}
$$

where $\mathcal{V}_{T_{A}(\varphi \otimes \varphi)}$ is defined in (4.3). Therefore, by Proposition 4.4, we have

$$
\begin{aligned}
\|R(f, g)\|_{\widetilde{M}_{1 / w}^{p_{3}^{\prime} p_{4}^{\prime} q_{3}^{\prime} q_{4}^{\prime}}} & =\left\|\mathcal{V}_{T_{A}(\varphi \otimes \varphi)} T_{A}(\bar{f} \otimes g)\right\|_{L_{1 / w}^{p_{3}^{\prime} p_{4}^{\prime} q_{3}^{\prime} q_{4}^{\prime}}} \\
& \leq\|f\|_{M_{w_{1}}^{p_{1} q_{1}}}\|g\|_{M_{1 / w_{2}}^{p_{2}^{\prime} q_{2}^{\prime}}} .
\end{aligned}
$$

To obtain (4.7) in the general case, that is $p_{1}, p_{2}, p_{3}, p_{4}, q_{1}, q_{2}, q_{3}, q_{4} \in[1, \infty]$ satisfy (4.6) but not necessarily (4.8), set

$$
\frac{1}{\widetilde{p}_{2}}=\frac{1}{p_{3}}+\frac{1}{p_{4}}-\frac{1}{p_{1}^{\prime}} \quad \text { and } \quad \frac{1}{\widetilde{q}_{2}}=\frac{1}{q_{3}}+\frac{1}{q_{4}}-\frac{1}{q_{1}^{\prime}} .
$$

Then it is easy to see that $\widetilde{p}_{2} \leq p_{2}, \widetilde{q}_{2} \leq q_{2}$ and $p_{1}, \widetilde{p}_{2}, p_{3}, p_{4}, q_{1}, \widetilde{q}_{2}, q_{3}, q_{4} \in[1, \infty]$ satisfy (4.6). Hence

$$
\left\|T_{\sigma} f\right\|_{M_{w_{2}}^{p_{2} q_{2}}} \leq C\left\|T_{\sigma} f\right\|_{M_{w_{2}}^{\widetilde{p}_{2} \widetilde{q}_{2}}} \leq\|f\|_{M_{w_{1}}^{p_{1} q_{1}}}\|\sigma\|_{\widetilde{M}_{w}^{p_{3} p_{4} q_{3} q_{4}}},
$$

for some $C>0$.

Proof of Theorem 4.1: Let $f \in \mathcal{S}\left(\mathbb{R}^{d}\right)$. Set

$$
\frac{1}{\widetilde{p}_{3}}=\frac{1}{p_{1}^{\prime}}+\frac{1}{p_{2}}-\frac{1}{p_{4}} \quad \text { and } \quad \frac{1}{\widetilde{q}_{3}}=\frac{1}{q_{1}^{\prime}}+\frac{1}{q_{2}}-\frac{1}{q_{4}} .
$$

Then it is easy to see that

$$
\widetilde{p}_{3} \geq p_{3}, \quad \widetilde{q}_{3} \geq q_{3} .
$$

Furthermore, $\left\{p_{1}, p_{2}, \widetilde{p}_{3}, p_{4}, q_{1}, q_{2}, \widetilde{q}_{3}, q_{4}\right\}$ satisfies (4.6), therefore there exist $C_{1}, C_{2}>0$ such that

$$
\begin{aligned}
\left\|T_{\sigma} f\right\|_{M_{w_{2}}^{p_{2} q_{2}}} & \leq C_{1}\|f\|_{M_{w_{1}}^{p_{1} q_{1}}}\|\sigma\|_{\widetilde{M}_{w}^{\tilde{\beta}_{3} p_{4} \tilde{q}_{3} q_{4}}} \\
& \leq C_{2}\|f\|_{M_{w_{1}}^{p_{1} q_{1}}}\|\sigma\|_{\widetilde{M}_{w}^{p_{3} p_{4} q_{3} q_{4}}} .
\end{aligned}
$$

\subsection{Proof of Corollary 1.4}

Let $1 \leq p, q \leq 2$. By Theorem 1.3, $T_{\sigma}: M^{p p^{\prime}} \rightarrow M^{q}$ is bounded. Using the bounded embeddings $M^{p} \subset \bar{L}^{p} \subset M^{p p^{\prime}}$ for all $1 \leq p \leq 2$ (for more details see [8]), it follows that $T_{\sigma}: L^{p}\left(\mathbb{R}^{d}\right) \rightarrow L^{q}\left(\mathbb{R}^{d}\right)$ is bounded. Similarly, using $M^{p p^{\prime}} \subset L^{p} \subset M^{p}$ for all $q \geq 2$, we can prove $T_{\sigma}: L^{p}\left(\mathbb{R}^{d}\right) \rightarrow L^{q}\left(\mathbb{R}^{d}\right)$ is bounded for $p, p_{3}, p_{4}, q, q_{3}, q_{4}$ satisfying the conditions of Corollary 1.4 .

\subsection{Proof of (1.4) implies (1.5), (1.6) in Theorem 1.3}

To show necessity of (1.5) and (1.6) in Theorem 1.3, we shall use two mixed $L^{p}$ norms on phase space, namely,

$$
\|F\|_{L^{p q}}=\left(\int\left(\int|F(x, \xi)|^{p} d x\right)^{q / p} d \xi\right)^{1 / q}
$$

and

$$
\|F\|_{\widetilde{L}^{p q}}=\left(\int\left(\int|F(x, \xi)|^{q} d \xi\right)^{p / q} d x\right)^{1 / p}
$$


for $p, q \in[1, \infty)$. For $p=\infty$ and/or $q=\infty$ we make the usual adjustment.

Similarly, we can define $\widetilde{M^{p q}}\left(\mathbb{R}^{d}\right)$ to be the space of all functions $f \in \mathcal{S}^{\prime}\left(\mathbb{R}^{d}\right)$ for which

$$
\|f\|_{\widetilde{M}^{p q}}=\left\|V_{\varphi} f\right\|_{\widetilde{L}^{p q}}<\infty
$$

where $\varphi \in \mathcal{S}\left(\mathbb{R}^{d}\right) \backslash\{0\}$. Note that it can be easily checked that

$$
\|f\|_{\widetilde{M}^{p q}}=\|\widehat{f}\|_{M^{q p}} .
$$

Below, we use an idea from the proof of Proposition 2.1 given in [22] to prove the following lemma.

Lemma 4.6. Let $K \subset \mathbb{R}^{2 d}$ be compact. Then

$$
\|\sigma\|_{\widetilde{M}^{p_{3} p_{4} q_{3} q_{4}}} \asymp\|\sigma\|_{\mathcal{F}^{q_{4} p_{4}}}, \quad \sigma \in \mathcal{S}^{\prime}\left(\mathbb{R}^{2 d}\right), \quad \operatorname{supp} \sigma \subset K .
$$

Proof. Choose $r>0$ with supp $\sigma \subseteq B_{r}^{2 d}(0)$, where

$$
B_{r}^{2 d}(0)=\left\{x \in \mathbb{R}^{2 d}:\|x\| \leq r\right\}
$$

is the Euclidean unit ball in $\mathbb{R}^{2 d}$ with center 0 , radius $r$ and Lebesgue measure $\left|B_{r}^{2 d}(0)\right|$. Let $\psi \in \mathcal{S}\left(\mathbb{R}^{2 d}\right)$ with supp $\psi \subset B_{r}^{2 d}(0)$. Then it is easy to see that

$$
\left|\widetilde{V}_{\psi} \sigma\right|(x, t, \xi, \nu)=\left|\sigma * M_{\nu,-t} \overline{\widetilde{\psi}}\right|(x, \xi),
$$

where

$$
\widetilde{\psi}(x, \xi)=\psi(-x,-\xi)
$$

Therefore, for fixed $t, \nu$ we have

$$
\begin{aligned}
\operatorname{supp}\left(\left|\widetilde{V}_{\psi} \sigma\right|(\cdot, t, \cdot, \nu)\right) & \subseteq \operatorname{supp}(\sigma)+\operatorname{supp}\left(M_{\nu,-t} \overline{\widetilde{\psi}}\right) \\
& \subseteq B_{r}^{2 d}(0)+B_{r}^{2 d}(0) \subseteq B_{2 r}^{2 d}(0) .
\end{aligned}
$$

Let $\xi \in B_{2 r}^{d}(0)$. Then by (4.9),

$$
\begin{aligned}
& \left\|\widetilde{V}_{\psi} \sigma(\cdot, t, \xi, \nu)\right\|_{L^{p_{3}\left(\mathbb{R}^{d}\right)}}^{p_{3}}=\int_{B_{2 r}^{d}(0)}\left|\widetilde{V}_{\psi} \sigma\right|^{p_{3}}(x, t, \xi, \nu) d x \\
& \quad \leq\left|B_{2 r}^{d}(0)\right|\left\|\widetilde{V}_{\psi} \sigma(\cdot, t, \xi, \nu)\right\|_{L^{\infty}}=\left|B_{2 r}^{d}(0)\right|\left\|\sigma * M_{\nu,-t} \overline{\widetilde{\psi}}(\cdot, \xi)\right\|_{L^{\infty}} \\
& \quad \leq\left|B_{2 r}^{d}(0)\right|\left\|\sigma * M_{\nu,-t} \overline{\widetilde{\psi}}\right\|_{L^{\infty}} \leq\left|B_{2 r}^{d}(0)\right|\left\|\mathcal{F}^{-1}\left(\widehat{\sigma} T_{\nu,-t} \overline{\widehat{\psi}}\right)\right\|_{L^{\infty}} \\
& \quad \leq\left|B_{2 r}^{d}(0)\right|\left\|\widehat{\sigma} T_{\nu,-t} \overline{\widehat{\psi}}\right\|_{L^{1}}=\left|B_{2 r}^{d}(0)\right|(|\widehat{\sigma}| *|\widehat{\psi}|)(-\nu, t)
\end{aligned}
$$

On the other hand, if $\xi \in \mathbb{R}^{d} \backslash B_{2 r}^{d}(0)$, then by (4.9),

$$
\left\|\widetilde{V}_{\psi} \sigma(\cdot, t, \xi, \nu)\right\|_{L^{p_{3}}}=0
$$

Therefore, (4.10) and (4.11) imply

$$
\begin{aligned}
& \|\sigma\|_{\widetilde{M}^{p_{3} p_{4} q_{3} q_{4}}}=\left\|\widetilde{V}_{\psi} \sigma\right\|_{L^{p_{3} p_{4} q_{3} q_{4}}} \\
& \quad \leq\left|B_{2 r}^{d}(0)\right|^{1 / p_{3}} \int\left(\int_{B_{2 r}^{d}(0)}\left(\int\left((|\widehat{\sigma}| *|\widehat{\psi}|(-\nu, t))^{p_{4}} d t\right)^{q_{3} / p_{4}} d \xi\right)^{q_{4} / q_{3}} d \nu\right)^{1 / q_{4}} \\
& \quad \leq\left|B_{2 r}^{d}(0)\right|^{\left(1 / p_{3}\right)+\left(1 / q_{3}\right)}\left(\int\left(\int(|\widehat{\sigma}| *|\widehat{\psi}|(-\nu, t))^{p_{4}} d t\right)^{q_{4} / p_{4}} d \nu\right)^{1 / q_{4}} \\
& \quad \leq\left|B_{2 r}^{d}(0)\right|^{\left(1 / p_{3}\right)+\left(1 / q_{3}\right)}\||\widehat{\sigma}| *|\widehat{\psi}|\|_{\widetilde{L}^{q_{4} p_{4}}} \\
& \quad \leq\left|B_{2 r}^{d}(0)\right|^{\left(1 / p_{3}\right)+\left(1 / q_{3}\right)}\|\widehat{\sigma}\|_{\widetilde{L}^{q_{4} p_{4}}}\|\widehat{\psi}\|_{\widetilde{L}^{11}} \leq C\|\widehat{\sigma}\|_{\widetilde{L}^{q_{4} p_{4}}} .
\end{aligned}
$$


Now, let $\psi \in C^{\infty}\left(\mathbb{R}^{2 d}\right)$ be compactly supported with $\psi \equiv 1$ on $B_{2 r}^{2 d}(0)$. Let $\chi_{B_{r}^{2 d}(0)}$ be the characteristic function on $B_{r}^{2 d}(0)$. Then using supp $\sigma \subseteq B_{r}^{2 d}(0)$, it follows that for all $x, t, \xi, \nu \in \mathbb{R}^{d}$,

$$
\begin{aligned}
& \chi_{B_{2 r}^{2 d}(0)}(x, \xi) \widetilde{V}_{\psi} \sigma(x, t, \xi, \nu) \\
& \quad=\chi_{B_{2 r}^{2 d}(0)}(x, \xi) \int_{B_{r}^{2 d}(0)} \sigma(\widetilde{x}, \widetilde{\xi}) e^{-2 \pi i(\widetilde{x} \nu-\widetilde{\xi} t)} \psi(\widetilde{x}-x, \widetilde{\xi}-\xi) d \widetilde{x} d \widetilde{\xi} \\
& \quad=\chi_{B_{2 r}^{2 d}(0)}(x, \xi) \int_{B_{r}^{2 d}(0)} \sigma(\widetilde{x}, \widetilde{\xi}) e^{-2 \pi i(\widetilde{x} \nu-\widetilde{\xi} t)} d \widetilde{x} d \widetilde{\xi} \\
& \quad=\chi_{B_{2 r}^{2 d}(0)}(x, \xi) \mathcal{F} \sigma(\nu,-t) .
\end{aligned}
$$

Hence,

$$
\begin{aligned}
& \|\sigma\|_{\widetilde{M}^{p_{3} p_{4} q_{3} q_{4}}}=\left\|\widetilde{V}_{\psi} \sigma\right\|_{L^{p_{3} p_{4} q_{3} q_{4}}} \geq\left\|\chi_{B_{2 r}^{2 d}(0)} \widetilde{V}_{\psi} \sigma\right\|_{L^{p_{3} p_{4} q_{3} q_{4}}} \\
& \quad=\left(\int\left(\int\left(\int\left(\int\left|\chi_{B_{2 r}^{2 d}(0)}(x, \xi) \mathcal{F} \sigma(\nu,-t)\right|^{p_{3}} d x\right)^{p_{4} / p_{3}} d t\right)^{q_{3} / p_{4}} d \xi\right)^{q_{4} / q_{3}} d \nu\right)^{1 / q_{4}} \\
& \quad=\left\|\chi_{B_{2 r}^{2 d}(0)}\right\|_{L^{p_{3} q_{3}}}\|\sigma\|_{\mathcal{F}_{\tilde{L}^{q_{4} p_{4}}}}
\end{aligned}
$$

which completes the proof.

Lemma 4.7. Let $\lambda>0$ and $\varphi_{\lambda}(x)=e^{-\pi \lambda|x|^{2}}$. Then for $\lambda \geq 1$,

$$
\left\|\varphi_{\lambda}\right\|_{M^{p q}} \asymp\left\|\varphi_{\lambda}\right\|_{\widetilde{M}^{p q}} \asymp \lambda^{-d / q^{\prime}}
$$

and

$$
\left\|\varphi_{\lambda^{-1}}\right\|_{M^{p q}} \asymp\left\|\varphi_{\lambda^{-1}}\right\|_{\widetilde{M}^{p q}} \asymp \lambda^{d / p} .
$$

The proof of Lemma 4.7 is an immediate corollary of Lemma 3.2 in [4] and is omitted here.

Lemma 4.8. Let $K \subset \mathbb{R}^{d}$ be compact. For $h \in C^{\infty}\left(\mathbb{R}^{d}\right)$ and $\lambda \geq 1$ set $h_{\lambda}(x)=h(x) e^{-\pi i \lambda|x|^{2}}$. Then for all $p, q \in[1, \infty]$,

$$
\left\|h_{\lambda}\right\|_{M^{p q}} \asymp\left\|\widehat{h}_{\lambda}\right\|_{L^{q}} \asymp \lambda^{d / q-d / 2}, \quad h \in C^{\infty}\left(\mathbb{R}^{d}\right), \quad \operatorname{supp} h \subset K .
$$

Lemma 4.8 is well known and its proof can be found in, for example, [5].

Lemma 4.9. Let $h_{1}, h_{2} \in \mathcal{S}\left(\mathbb{R}^{d}\right)$ and

$$
\eta(t, \nu)=e^{-2 \pi i t \nu} h_{2}(t) \widehat{h}_{1}(\nu), \quad t, \nu \in \mathbb{R}^{d} .
$$

If $\sigma=\widetilde{\mathcal{F}} \eta$. Then we have

$$
\sigma(x, \xi)=\left(M_{\xi} h_{2} * h_{1}\right)(x)
$$

and

$$
T_{\sigma} f=\left(h_{1} f\right) * h_{2}, \quad f \in \mathcal{S}\left(\mathbb{R}^{d}\right)
$$

Moreover,

$$
\|\sigma\|_{\widetilde{M}^{p_{3} p_{4} q_{3} q_{4}}}=\left\|h_{1}\right\|_{M^{p_{3} q_{4}}}\left\|h_{2}\right\|_{M^{p_{4} q_{3}}} .
$$

Proof. Clearly, (4.12) and (4.13) hold. Now, let $\varphi$ be any nonzero real valued Schwartz function on $\mathbb{R}^{d}$. Let

$$
\psi(t, \nu)=\varphi(t) \varphi(\nu) e^{-2 \pi i t \nu}
$$


and define

$$
\widetilde{\psi}(x, \xi)=\widetilde{\mathcal{F}} \psi(-x,-\xi)
$$

Then

$$
\begin{aligned}
\left|\widetilde{V}_{\widetilde{\psi}} \sigma(x, t, \xi, \nu)\right| & =\left|\left(\sigma, M_{\nu,-t} T_{x, \xi} \widetilde{\psi}\right)\right| \\
& =\left|\left(\widetilde{\mathcal{F}} \eta, \widetilde{\mathcal{F}}\left(T_{-t, \nu} M_{-\xi, x} \psi\right)\right)\right|
\end{aligned}
$$

Now since $\widetilde{\mathcal{F}}$ is a unitary operator, it follows that

$$
\begin{aligned}
& \left|\left(\widetilde{V}_{\widetilde{\psi}} \sigma\right)(x, t, \xi, \nu)\right|=\left|\left(\eta, T_{-t, \nu} M_{-\xi, x} \psi\right)\right| \\
& \quad=\left|\iint \eta(\widetilde{t}, \widetilde{\nu}) e^{2 \pi i \xi(\widetilde{t}+t)} e^{-2 \pi i x(v-\widetilde{\nu})} \bar{\psi}(t+\widetilde{t}, \widetilde{\nu}-\nu) d \widetilde{t} d \widetilde{\nu}\right| \\
& \quad=\left|\iint \widehat{h}_{1}(\widetilde{\nu}) h_{2}(\widetilde{t}) \varphi(\widetilde{\nu}-\nu) \varphi(\widetilde{t}+t) e^{-2 \pi i \widetilde{\nu}(x-t)} e^{2 \pi i \widetilde{t}(\nu-\xi)} d \widetilde{t} d \widetilde{\nu}\right| \\
& \quad=\left|\left(V_{\varphi} \widehat{h}_{1}\right)(\nu, x-t)\right|\left|\left(V_{\varphi} h_{2}\right)(-t, \nu-\xi)\right| .
\end{aligned}
$$

Hence,

$$
\|\sigma\|_{\widetilde{M}^{p_{3} p_{4} q_{3} q_{4}}}=\left\|h_{1}\right\|_{M^{p_{3} q_{4}}}\left\|h_{2}\right\|_{M^{p_{4} q_{3}}} .
$$

Similarly, we can prove the following.

Lemma 4.10. Let Let $h_{1}, h_{2} \in \mathcal{S}\left(\mathbb{R}^{d}\right)$ and $\sigma=h_{1} \otimes \widehat{h}_{2}$. Then

$$
T_{\sigma} f=h_{1} \cdot\left(h_{2} * f\right), \quad f \in \mathcal{S}\left(\mathbb{R}^{d}\right)
$$

and

$$
\left\|h_{1} \otimes \widehat{h}_{2}\right\|_{\widetilde{M}^{p_{3} p_{4} q_{3} q_{4}}}=\left\|h_{1}\right\|_{M^{p_{3} q_{4}}}\left\|\widehat{h}_{2}\right\|_{\widetilde{M}^{q_{3} p_{4}}} .
$$

Proof of (1.4) implies (1.5) and (1.6) in Theorem 1.3: Let $h \in C^{\infty}\left(\mathbb{R}^{d}\right)$ be chosen with compact support and $h(0)=1$ and $h(x) \geq 0$ for all $x \in \mathbb{R}^{d}$. Then for any $\lambda \geq 1$, we define $h_{\lambda}$ and $\sigma_{\lambda}$ respectively by

$$
h_{\lambda}(x)=h(x) e^{-\pi i \lambda|x|^{2}} .
$$

and

$$
\sigma_{\lambda}(x, \xi)=h \otimes h_{\lambda}(x, \xi)=h(x) h_{\lambda}(\xi) .
$$

Let $f_{\lambda}=\mathcal{F}^{-1} \overline{h_{\lambda}}$. Then $f_{\lambda} \in \mathcal{S}\left(\mathbb{R}^{d}\right)$ and

$$
T_{\sigma_{\lambda}} f_{\lambda}(x)=\int e^{2 \pi i x \xi} h(x)|h(\xi)|^{2} d \xi .
$$

So, $T_{\sigma_{\lambda}} f_{\lambda}$ is independent of $\lambda$. Since $\sigma_{\lambda}$ has compact support, by Lemma 4.6 and Lemma 4.8

$$
\begin{aligned}
\left\|\sigma_{\lambda}\right\|_{\widetilde{M}^{p_{3} p_{4} q_{3} q_{4}}} & \asymp\left\|\mathcal{F} \sigma_{\lambda}\right\|_{\widetilde{L}^{q_{4} p_{4}}} \\
& =\|\widehat{h}\|_{L^{q_{4}}\left(\mathbb{R}^{d}\right)}\left\|\widehat{h}_{\lambda}\right\|_{L^{p_{4}}\left(\mathbb{R}^{d}\right)} \\
& \asymp \lambda^{\left(d / p_{4}\right)-(d / 2)} .
\end{aligned}
$$

Moreover, by Lemma 2.1 and Lemma 4.8, since $\mathcal{F} f_{\lambda}$ has compact support,

$$
\left\|f_{\lambda}\right\|_{M^{p_{1} q_{1}}\left(\mathbb{R}^{d}\right)}=\left\|f_{\lambda}\right\|_{L^{p_{1}\left(\mathbb{R}^{d}\right)}} \asymp \lambda^{\left(d / p_{1}\right)-(d / 2)} .
$$


Hence by (1.4), (4.14) and (4.15), there exists $C>0$ such that for all $\lambda \geq 1$

$$
\left\|T_{\sigma} f_{\lambda}\right\|_{M^{p_{2} q_{2}\left(\mathbb{R}^{d}\right)}} \leq C \lambda^{\left(d / p_{4}\right)+\left(d / p_{1}\right)-d} .
$$

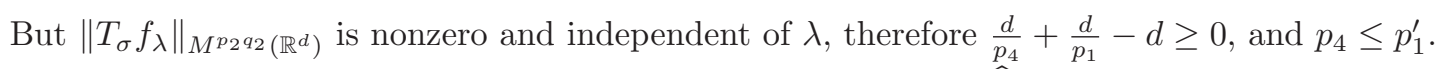

To prove $q_{4} \leq q_{1}^{\prime}$, we let $h_{1}=\bar{f}=h_{\lambda}$ and $h_{2} \in \mathcal{S}\left(\mathbb{R}^{d}\right)$ be such that $\widehat{h}_{2}$ is compactly supported, independent of $\lambda$ and

$$
\left\|\left(h_{1} f\right) * h_{2}\right\|_{L^{p_{2}\left(\mathbb{R}^{d}\right)}} \neq 0 .
$$

Let $\sigma=\widetilde{\mathcal{F}} \eta$ where

$$
\eta(t, \nu)=\widehat{h}_{1}(\nu) h_{2}(t) e^{-2 \pi i t \nu}
$$

Then by Lemma 4.9 and (1.4)

$$
\left\|\left(h_{1} f\right) * h_{2}\right\|_{L^{p_{2}\left(\mathbb{R}^{d}\right)}} \leq C\left\|\widehat{h}_{1}\right\|_{L^{q_{4}}\left(\mathbb{R}^{d}\right)}\left\|h_{2}\right\|_{L^{p_{4}}\left(\mathbb{R}^{d}\right)}\|\widehat{f}\|_{L^{q_{1}}\left(\mathbb{R}^{d}\right)},
$$

for some constant $C>0$. So, by Lemma 4.8 for all $\lambda \geq 1$

$$
\left\|\left(h_{1} f\right) * h_{2}\right\|_{L^{p_{2}\left(\mathbb{R}^{d}\right)}} \leq C \lambda^{\left(d / q_{4}\right)-(d / 2)} \lambda^{\left(d / q_{1}\right)-(d / 2)},
$$

but $\left\|\left(h_{1} f\right) * h_{2}\right\|_{L^{p_{2}\left(\mathbb{R}^{d}\right)}}$ is nonzero and independent of $\lambda$, therefore $\left(d / q_{4}\right)+\left(d / q_{1}\right)-d \geq 0$ and, hence, $q_{4} \leq q_{1}^{\prime}$.

Now, let $h_{1}=f=\varphi_{\lambda}$ and $h_{2}=\varphi_{\lambda^{-1}}$, where $\varphi_{\lambda}$ and $\varphi_{\lambda^{-1}}$ are defined in Lemma 4.7. If we let $\sigma=h_{1} \otimes h_{2}$. Then by Lemma 4.7 and Lemma 4.10 , for $\lambda \geq 1$ we have

$$
\|\sigma\|_{\widetilde{M}^{p_{3} p_{4} q_{3} q_{4}\left(\mathbb{R}^{2 d}\right)}} \asymp \lambda^{d / q_{3}-d / q_{4}^{\prime}}
$$

and $\|f\|_{M^{p_{1} q_{1}\left(\mathbb{R}^{d}\right)}} \asymp \lambda^{-d / q_{1}^{\prime}}$. On the other hand $T_{\sigma} f$ is also a Gaussian function and it can be easily checked that

$$
\left\|T_{\sigma} f\right\|_{M^{p_{2} q_{2}\left(\mathbb{R}^{d}\right)}} \asymp \lambda^{-d / q_{2}^{\prime}} .
$$

Therefore by (1.4)

$$
\lambda^{d / q_{3}-d / q_{4}^{\prime}-d / q_{1}^{\prime}+d / q_{2}^{\prime}} \geq 1
$$

for all $\lambda \geq 1$. Hence, we get

$$
\frac{1}{q_{1}^{\prime}}+\frac{1}{q_{2}} \leq \frac{1}{q_{3}}+\frac{1}{q_{4}}
$$

Similarly, by letting $h_{1}=f=\varphi_{\lambda^{-1}}$ and $h_{2}=\varphi_{\lambda}$, we get

$$
\frac{1}{p_{1}^{\prime}}+\frac{1}{p_{2}} \leq \frac{1}{p_{3}}+\frac{1}{p_{4}} \text {. }
$$

Again assume $\sigma$ has the form given in Lemma 4.9. Let $h(x)=f(x)=e^{-\pi|x|^{2} / 2}$ and $h_{2}=\varphi_{\lambda^{-1}}$. Then $T_{\sigma}$ is also a Gaussian function, moreover by Lemma 4.7 and (1.4) for all $\lambda \geq 1$

$$
\lambda^{d / p_{4}-d / p_{2}} \geq C
$$

for some $C>0$. Hence $p_{4} \leq p_{2}$.

To prove $q_{4} \leq q_{2}$, we let

$$
\sigma(x, \xi)=e^{2 \pi i x \xi} h_{1}(x) h_{2}(\xi),
$$

where $h_{1}$ and $h_{2}$ are compactly supported Schwartz functions on $\mathbb{R}^{d}$. Then $\sigma$ is compactly supported and therefore by Lemma 4.6 ,

$$
\|\sigma\|_{\widetilde{M}^{p_{3} p_{4} q_{3} q_{4}\left(\mathbb{R}^{2 d}\right)}}=\|\mathcal{F} \sigma\|_{L^{p_{4} q_{4}}\left(\mathbb{R}^{2 d}\right)} .
$$


On the other hand, by an easy calculation, we have

$$
|\mathcal{F} \sigma|(\nu, t)=\left|V_{h_{1}} \widehat{h}_{2}\right|(t, \nu)=\left|V_{\widehat{h_{2}}} \overline{h_{1}}\right|(t, \nu) .
$$

Therefore,

$$
\|\sigma\|_{\widetilde{M}^{p_{3} p_{4} q_{3} q_{4}\left(\mathbb{R}^{2 d}\right)}} \leq C_{h_{2}}\left\|\widehat{h}_{1}\right\|_{L^{q_{4}}\left(\mathbb{R}^{d}\right)},
$$

and

$$
\|\sigma\|_{\widetilde{M}^{p_{3} p_{4} q_{3} q_{4}\left(\mathbb{R}^{2 d}\right)}} \leq C_{h_{1}}\left\|\widehat{h}_{2}\right\|_{L^{p_{4}\left(\mathbb{R}^{d}\right)}},
$$

where $C_{h_{1}}$ and $C_{h_{2}}$ are positive constants depending on $h_{1}$ and $h_{2}$ respectively. Let $h_{1}=h_{\lambda}$ and $h_{2}$ be any compactly supported function and $f$ be a Schwartz function on $\mathbb{R}^{d}$ and both $h_{2}$ and $\widehat{f}$ be independent of $\lambda$ such that $\left(h_{2}, \overline{\widehat{f}}\right) \neq 0$. Then

$$
\begin{aligned}
\left\|T_{\sigma} f\right\|_{M^{p_{2} q_{2}\left(\mathbb{R}^{d}\right)}} & =\left\|h_{1}\right\|_{M^{p_{2} q_{2}\left(\mathbb{R}^{d}\right)}}\left|\left(h_{2}, \overline{\widehat{f}}\right)\right| \\
& =\left|\left(h_{2}, \overline{\widehat{f}}\right)\right|\left\|\widehat{h}_{1}\right\|_{L^{q_{2}\left(\mathbb{R}^{d}\right)}} \asymp \lambda^{\left(d / q_{2}\right)-(d / 2)},
\end{aligned}
$$

and by (4.16)

Hence, (4.17) and (1.4) imply

$$
\|\sigma\|_{\widetilde{M}^{p_{3} p_{4} q_{3} q_{4}\left(\mathbb{R}^{2 d}\right)}} \leq C_{h_{2}} \lambda^{\left(d / q_{4}\right)-(d / 2)}
$$

$$
\lambda^{\left(d / q_{4}\right)-\left(d / q_{2}\right)} \geq C,
$$

where $C>0$ is independent of $\lambda \geq 1$. Hence $\left(d / q_{4}\right)-\left(d / q_{2}\right) \geq 0$ which implies that $q_{4} \leq q_{2}$.

\section{References}

[1] A. Benedek, R. Panzone. The Space L ${ }^{p}$, with Mixed Norm. Duke Math. J. 28 (1961), 301-324.

[2] S. Bishop. Mixed modulation spaces and their application to pseudodifferential operators. J. Math. Anal. Appl. 363 (2010) 1, 255-264.

[3] V. Catană, S. Molahajloo, M. W. Wong. $L^{p}$-Boundedness of Multilinear Pseudo-Differential Operators, in PseudoDifferential Operators: Complex Analysis and Partial Differential Equations. Operator Theory: Advances and Applications. 205, Birkhäuser, 2010, 167-180.

[4] E. Cordero, F. Nicola. Metaplectic Representation on Wiener Amalgam Spaces and Applications to the Schrödinger Equation. J. Funct. Anal. 254 (2008), 506-534.

[5] E. Cordero, F. Nicola. Pseudodifferential Operators on $L^{p}$, Wiener Amalgam and Modulation Spaces. Int. Math. Res. Notices 10 (2010), 1860-1893.

[6] F. Concetti, J. Toft. Trace Ideals for Fourier Integral Operators with Non-Smooth Symbols, in Pseudo-Differential Operators: Partial Differential Equations and Time Frequency Analysis. Fields Institute Communications, 52 (2007), 255-264.

[7] W. Czaja. Boundedness of Pseudodifferential Operators on Modulation Spaces. J. Math. Anal. Appl. 284(1) (2003), 389-396.

[8] H. G. Feichtinger. Atomic Characterization of Modulation Spaces through the Gabor-Type Representations. Rocky Mountain J. Math. 19 (1989), 113-126.

[9] H. G. Feichtinger. On a New Segal Algebra. Monatsh. Math. 92 (1981), 269-289.

[10] H. G. Feichtinger, K. Gröchenig. Banach Spaces Related to Integrable Group Representations and Their Atomic Decompositions I. J. Funct. Anal. 86 (1989), 307-340.

[11] H. G. Feichtinger, K. Gröchenig. Banach Spaces Related to Integrable Group Representations and Their Atomic Decompositions II. Monatsh. Math. 108 (1989), 129-148.

[12] H. G. Feichtinger, K. Gröchenig. Gabor Wavelets and the Heisenberg Group: Gabor Expansions and Short Time Fourier Transform from the Group Theoretical Point of View, in Wavelets: a tutorial in theory and applications. Academic Press, Boston, 1992.

[13] H. G. Feichtinger, K. Gröchenig. Gabor Frames and Time-Frequency Analysis of Distributions. J. Funct. Anal. 146 (1997), 464-495.

[14] K. Gröchenig. Foundation of Time-Frequency Analysis. Brikhäuser, Boston, 2001.

[15] K. Gröchenig, C. Heil. Counterexamples for Boundedness of Pseudodifferential Operators. Osaka J. Math. 41 (3) (2004), 681-691.

[16] K. Gröchenig, C. Heil. Modulation Spaces and Pseudodifferential Operators. Integr. Equat. Oper. th. 34 (4) (1999), 439-457. 
[17] Y. M. Hong, G. E. Pfander. Irregular and multi-channel sampling of operators. Appl. Comput. Harmon. Anal. 29 (2) (2010), 214-231.

[18] L. Hörmander. The Analysis of Linear Partial Differential Operators I. Second Edition, Springer-Verlag, Berlin, 1990.

[19] L. Hörmander. The Weyl Calculus of Pseudodifferential Operators. Comm. Pure Appl. Math. 32 (1979), $360-444$.

[20] I. L. Hwang, R. B. Lee. $L^{p}$-Boundedness of Pseudo-Differential Operators of Class $S_{0,0}$. Trans. Amer. Math. Soc. 346 (2) (1994), 489-510.

[21] H. Kumano-Go. Pseudo-Differential Operators. Translated by Hitoshi Kumano-Go, Rémi Vaillancourt and Michihiro Nagase, MIT Press, 1982.

[22] K. A. Okoudjou. A Beurling-Helson Type Theorem for Modulation Spaces. J. Func. Spaces Appl. 7 (1) (2009), $33-41$.

[23] G. E. Pfander, D. Walnut. Operator Identification and Feichtinger's Algebra. Sampl. Theory Signal Image Process. 5 (2) (2006), 151-168.

[24] G. E. Pfander. Sampling of Operators. arxiv: 1010.6165.

[25] J. Sjöstrand. An Algebra of Pseudodifferential Operators. Math. Res. Lett. 1 (2) (1994), 185-192.

[26] J. Sjöstrand. Wiender Type Algebras of Pseudodifferential Operators, in Séminaire Équations aux dérivées Partielles. 1994-1995, exp. 4, 1-19.

[27] J. Toft. Continuity Properties for Modulation Spaces, with Applications to Pseudo-Differential Calculus I. J. Funct. Anal. 207 (2004), 399-429

[28] J. Toft. Continuity Properties for Modulation Spaces, with Applications to Pseudo-Differential Calculus II. Ann. Glob. Anal. Geom. 26 (2004), 73-106.

[29] J. Toft. Fourier Modulation Spaces and Positivity in Twisted Convolution Algebra. Integral Transforms and Special Functions, 17 nos. 2-3 (2006), 193-198.

[30] J. Toft. Pseudo-Differential Operators with Smooth Symbols on Modulation Spaces. CUBO. 11 (2009), 87-107.

[31] J. Toft, S. Pilipovic, N. Teofanov. Micro-Local Analysis in Fourier Lebesgue and Modulation Spaces. Part II, J. Pseudo-Differ. Oper. Appl. 1 (2010), 341-376.

[32] M. W. Wong. An Introduction to Pseudo-Differential Operators. Second Edition, World Scientific, 1999.

[33] M. W. Wong. Fredholm Pseudo-Differential Operators on Weighted Sobolev Spaces. Ark. Mat. 21 (2) (1983), $271-282$.

[34] M. W. Wong. Weyl Transforms. Springer-Verlag, 1998. 